\title{
Ferromagnetic materials in the zinc-blende structure
}

\author{
B. Sanyal, L. Bergqvist and O. Eriksson \\ Department of Physics, Uppsala University, Uppsala, Sweden
}

(Dated: August 28, 2018)

\begin{abstract}
New materials are currently sought for use in spintronics applications. Ferromagnetic materials with half metallic properties are valuable in this respect. Here we present the electronic structure and magnetic properties of binary compounds consisting of $3 \mathrm{~d}$ transition metals and group $\mathrm{V}$ elements viz. $\mathrm{P}, \mathrm{Sb}$ and $\mathrm{As}$ in the zinc-blende structure. We demonstrate that compounds of $\mathrm{V}, \mathrm{Cr}$ and Mn show half metallic behavior for appropriate lattice constants. By comparing the total energies in the ferromagnetic and antiferromagnetic structures, we have ascertained that the ferromagnetic phase is stable over the antiferromagnetic one. Of the different compounds studied, the Cr based systems exhibit the strongest interatomic exchange interactions, and are hence predicted to have the highest critical temperatures. Also, we predict that VAs under certain growth conditions should be a semiconducting ferromagnet. Moreover, critical temperatures of selected half metallic compounds have been estimated from mean field theory and Monte Carlo simulations using parameters obtained from a ab-initio non-collinear, tight binding linearized muffin-tin orbital method. ¿From a simple model, we calculate the reflectance from an ideal MnAs/InAs interface considering the band structures of MnAs and InAs. Finally we present results on the relative stabilities of MnAs and $\mathrm{CrSb}$ compounds in the NiAs and zinc-blende structures, and suggest a parameter space in substrate lattice spacings for when the zinc-blende structure is expected to be stable.
\end{abstract}

PACS numbers: 71.20.Be,75.50.Pp,71.70.Gm

\section{INTRODUCTION}

For magnetoelectronic devices, highly spin polarized ferromagnets have a large potential in applications [1]. Half metallic ferromagnets (HMF) are the most desirable materials in this respect. These materials have one spin channel that is metallic, while the other spin channel has a gap at the Fermi level. Hence, $100 \%$ spin polarization of the conducting electrons is expected from these materials. Based on band-structure calculations, de Groot et al. 2] first predicted half metallic behavior of $\mathrm{C}_{b}$ type Heusler alloys of NiMnSb and PtMnSb. Since then, there have been predictions of many half metallic materials. A practical aspect of these materials is the recent breakthrough utilizing the spin of electrons in existing semiconductor electronic devices. To manipulate spins of electrons inside the semiconductors, one first needs to inject spins inside the semiconductors. Spin injection can be done either from ferromagnetic metals or from diluted magnetic semiconductors (DMS) doped with magnetic ions. Ohno et al. 3] successfully injected spin polarized holes from a p-type III-V DMS to a semiconductor. From theoretical calculations of magnetotransport, Egues [4] proposed a spin filter for spin-dependent optoelectronics applications. Later on, Fiederling et al. [5] successfully injected spin polarized electrons from an n-type IIVI semiconductor. However, according to Schmidt et al. [6], the spin injection from a metal to a semiconductor is practically impossible due to the huge conductivity mismatch between a metal and a semiconductor. The use of a DMS is instead rather advantageous. Mn doped GaAs is a prototype of this class of systems but the highest available Curie temperature is only $110 \mathrm{~K}$ 7] due to the limited solubility of Mn in GaAs. The use of other half metallic systems e.g. $\mathrm{CrO}_{2}$ and $\mathrm{La}_{0.7} \mathrm{Sr}_{0.3} \mathrm{MnO}_{3}$ is also restricted due to lower Curie temperature. Very recently, there have been reports on room temperature ferromagnetism in Mn doped GaP compounds [8]. Hence, the quest for new half metallic materials with room temperature ferromagnetism continues.

Recently, there have been efforts to grow binary compounds in the zinc-blende structure using molecular beam epitaxy. One of the advantages of these materials is that the zinc-blende structure is maintained when grown on a zinc-blende semiconductor substrate. Akinaga et al. 9] grew CrAs on GaAs substrates and found $\mathrm{CrAs}$ to be a ferromagnet having a Curie temperature higher than 400 $\mathrm{K}$. The experimental results were complemented by $a b$ initio electronic structure calculations showing that the magnetic moment was $3 \mu_{B}$. Also $\mathrm{CrSb}$ was grown in the zinc-blende structure 10]. These binary compounds do not exist naturally in the zinc-blende structure but the non equilibrium growth by MBE has facilitated the creation of these compounds with novel magnetic properties.

Sanvito and Hill 11] examined the ground state properties of MnAs in a NiAs and a zinc-blende structures by first principles pseudopotential calculations. Shirai 12 performed electronic structure calculations for the zincblende compounds of transition metal (TM) and As. The early TM-As compounds were shown to be ferromagnets and the later compounds were antiferromagnets. Also, electronic structure calculations have been performed for different crystal structures of $\mathrm{MnP}, \mathrm{MnAs}$ and $\mathrm{MnSb}$ by Continenza et al. 13. Galanakis 14 examined the half metallicity of the bulk and surface of zinc-blende CrAs in various lattice constants by electronic structure calculations. A full potential screened Korringa-Kohn-Rostoker method was used. Galanakis concluded that a $\mathrm{Cr}$ ter- 
minated CrAs (001) surface remains half metallic both for the experimental lattice constants of GaAs and InAs. On the contrary, an As terminated surface loses its half metallicity due to the presence of the surface dangling bond states.

The motivation of this work is to design new materials in a crystal structure that is suitable for spin electronics applications and provide a guideline for experimental work that attempt to grow these materials. The paper is organized as follows : In section 'Computational details', we describe in detail the computational part. In the results section, a subsection 'Electronic structure and magnetism' describes the electronic structure and magnetism of the binary compounds considered followed by the discussions about the interatomic exchange interactions and critical temperatures in some of the systems studied. Finally, structural stabilities of $\mathrm{MnAs}$ and $\mathrm{CrSb}$ compounds in various structural phases are presented.

\section{COMPUTATIONAL DETAILS}

A zinc-blende structure has two fcc sublattices displaced from each other along the body diagonal by a vector $\left(\frac{1}{4} \frac{1}{4} \frac{1}{4}\right)$. For calculations in the ferromagnetic phase, the unit cell used in the calculations is the same as in the fcc lattice and has $1 \mathrm{TM}$ at the (000) and the group V atom at the $\left(\frac{1}{4} \frac{1}{4} \frac{1}{4}\right)$ position. Calculations have been performed by an ab-initio plane wave pseudopotential code (VASP) [15]. Pseudopotentials generated using the Projector augmented wave (PAW) [16] method were used throughout the calculations. These pseudopotentials are suitable for transition metals (TM) and the calculations yield accurate results [17]. A kinetic energy cut-off of 600 $\mathrm{eV}$ was used for the plane waves included in the basis set. Perdew-Wang GGA (generalized gradient approximation) exchange -correlation [18] was used. According to the calculations of Continenza et al. 13], GGA is essential for obtaining accurate equilibrium volume and magnetic moments. A 20x20x20 k-points grid was used in the Monkhorst Pack scheme [19] which yielded $770 \mathrm{k}$ points in the irreducible Brillouin zone and subsequently used for the Brillouin zone integration within the tetrahedron method. Local properties such as local density of states and local magnetic moments were calculated by projecting the wave functions into spherical harmonics as described in ref. 20]. The values of the experimental lattice constants used in these calculations were taken from Ashcroft and Mermin 21]. Calculations were done for the TM-V compounds for the lattice constants of the III-V semiconductors having the same Group V element. In summary, we considered lattice constants of GaAs, GaP, $\mathrm{GaSb}$, InAs, InP and InSb compounds which covers a wide range (5.45 $\AA$ for $\mathrm{GaP}$ to $6.48 \AA$ for $\mathrm{InSb}$ ).

The pseudopotential calculations have been complemented 22] with calculations based on non-collinear tight-binding linear muffin-tin orbital method (TBLMTO-ASA) 23] including combined correction terms in the one-electron Hamiltonian 24]. The generalized gradient approximation (GGA) were used for the exchangecorrelation potential 25]. Equal Wigner-Seitz sphere radii were used for all atoms, as well as for the empty spheres representing the two types of interstitial sites in the zinc-blende structure. A dense $27 \times 27 \times 27$ k-point grid was used in the Brillouin zone integration and to speed up convergence each energy eigenvalue was associated with a Gaussian of width $85 \mathrm{meV}$. Spin wave excitations have been studied using the frozen-magnon approach [26, 27, 28]. Then the total energy $E(\mathbf{q}, \theta)$ is calculated for spiral magnetic structures where each magnetic moment is defined by the Euler angles

$$
\theta_{i}=\text { const } ; \phi_{i}=\mathbf{q} \cdot \mathbf{R}_{i}
$$

where $\theta_{i}$ and $\phi_{i}$ are the polar and azimuthal angles, $\mathbf{R}_{i}$ the position of ion $i$ and $\mathbf{q}$ is the spiral propagation vector. Spin spirals possess a generalized translational symmetry [29], which allow for calculations in the chemical unit cell without need for a supercell. It can be shown, that the true spin wave excitation energies are related to the spiral energies through the relation

$$
\omega(\mathbf{q})=\frac{4}{M} \frac{\Delta E(\mathbf{q}, \theta)}{\sin ^{2} \theta},
$$

where $\Delta E(\mathbf{q}, \theta)=E(\mathbf{q}, \theta)-E(0, \theta)$. The last equation is valid for one atom per cell. Thus the formula is correct if one consider the magnetic response from the transition metal only and neglect the small induced magnetic moment on the group $\mathrm{V}$ element. In our calculations, we use an angle $\theta=20^{\circ}$ and the spiral energies were obtained by employing the magnetic force theorem. We tested other angles as well as self-consistent calculations and found that the differences in total energy is within a few percent.

In order to obtain thermodynamic properties, the spin wave excitation energies were mapped to an effective Heisenberg Hamiltonian of classical spins

$$
H=-\sum_{i \neq j} J_{i j} \mathbf{e}_{i} \cdot \mathbf{e}_{j}
$$

where $J_{i j}$ are exchange interactions between the transition metal atoms. The critical temperatures $T_{c}$ were estimated both from mean field theory 21] (MFA) and Monte Carlo simulations (MC). For the MC simulations, we used the standard single flip Metropolis algorithm and the critical temperature were obtained from the 'cumulant crossing method' 30]. In this method, the reduced fourth order cumulant of the order parameter (magnetization) $U_{L}$, defined as

$$
U_{L}=1-\frac{\left\langle M^{4}\right\rangle}{3\left\langle M^{2}\right\rangle^{2}}
$$


is calculated for different sizes of the simulation box. The curves of $U_{L}$ have a common intersection point at a fixed point $U^{*}$, which corresponds to the critical point. Hence, a value of $T_{c}$ is obtained from the intersection point of $U_{L}$ for different lattice sizes.

\section{RESULTS AND DISCUSSIONS}

\section{A. Electronic structure and magnetism}

The zinc-blende lattice introduces a tetrahedral crystal field under which the transition metal atomic $d$-states are split into triply degenerate $t_{2}$ and doubly degenerate $e$ states. $e$ states lie lower in energy than $t_{2}$ states in a tetrahedral crystal field in contrast to the octahedral crystal field. The $t_{2}$ states hybridize with the anion $p$-states. As a result, bonding and antibonding states appear. Also, depending on the exchange splitting, the bonding and antibonding states may lie in different positions for different spin channels. $e$ states are of nonbonding character and do not take part in the hybridization.

In Fig. 1(a-f), we present the spin resolved DOSs of $\mathrm{V}, \mathrm{Cr}$ and Mn compounds having the lattice constants of GaAs, InAs, GaSb, InSb, GaP and InP. In figs. (a), (b), (c), (d) and (f), all the compounds show half metallic behavior since there is no state at the Fermi level for the spin down DOS. The only exception is the case depicted in fig. 1(e) which doesn't exhibit half metallic ferromagnetism as the Fermi level cuts both the spin bands. For the lower lattice constant of GaP, the $p-d$ overlap and hybridization of the spin down states broadens the band to cut the Fermi level. Also, as the width of the gap in the spin down DOS is quite large, the Fermi level stays within the gap with the variation in the lattice constant. It is worthy to be mentioned here that these compounds can be regarded to be the derivatives of diluted magnetic semiconductors, e.g. $\mathrm{Mn}_{x} \mathrm{Ga}_{1-x}$ As for a Mn doped GaAs system having $x=1$. Several first principles calculations [31, 32, 33, 34] confirmed that the total spin magnetic moment of Mn doped GaAs for various concentrations of $\mathrm{Mn}$ is $4 \mu_{B}$ /cell with a half metallic nature. It was also shown [17] that $\mathrm{Cr}$ doped GaAs is a half metallic ferromagnet with a total spin magnetic moment of $3 \mu_{B} /$ cell. We find here a similar trend for the extreme limit at a high TM concentration. A systematic variation in the DOSs of $\mathrm{V}, \mathrm{Cr}$ and $\mathrm{Mn}$ is seen. As the number of valence electrons increases by going from $\mathrm{V}$ to $\mathrm{Mn}$, the main features of the DOSs shift to lower energies relative to the Fermi level. This can be seen from figs. 1 (b) and 1 (f). The exchange splitting is maximum for $\mathrm{Mn}$ and here one finds a maximum separation between the spin up and spin down bands.

A close inspection of Fig. 1(a) reveals that VAs behaves like a magnetic semiconductor for the lattice constant of GaAs, i.e. the Fermi level passes through a gap for both spin up and spin down channels. We varied

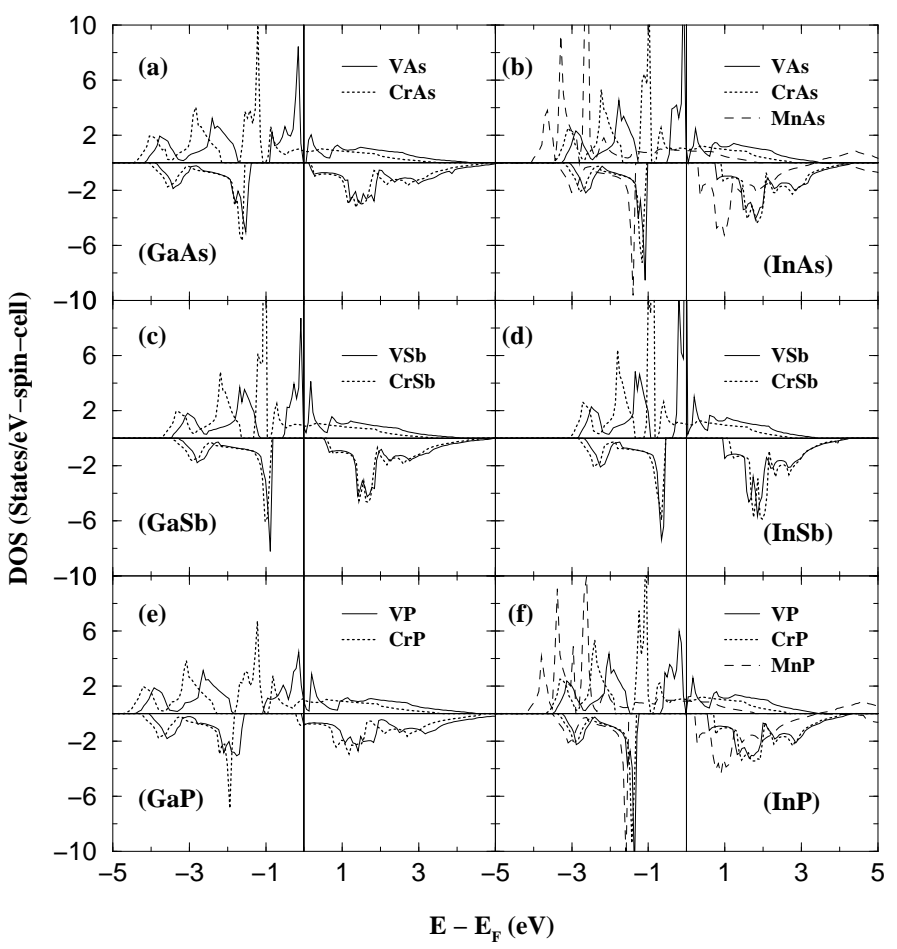

FIG. 1: Spin resolved total (sum over atoms and orbitals) density of states (DOS) calculated for the unit cell. In (a), we show the DOSs for VAs and CrAs in the GaAs lattice constant, in (b), VAs, CrAs and MnAs in the InAs lattice constant, in (c), VSb and CrSb in the GaSb lattice constant, in (d), VSb and CrSb in the InSb lattice constant, in (e), VP and $\mathrm{CrP}$ in the GaP lattice constant and in (f), VP, CrP and $\mathrm{MnP}$ in the InP lattice constant. The energies are shifted with respect to the corresponding Fermi levels and energies are in electron volts.

the lattice constant of VAs around the GaAs lattice constant and found that the small gap is sensitive to the lattice spacing. Only in a very small region around the GaAs lattice constant, do we find that the gap persists. This is an interesting result since it is one of quite few ferromagnetic semiconductors found in a stoichiometric composition. In addition, it is to be noted that unlike CrAs, MnAs doesn't show half metallic behavior for the GaAs lattice constant. It is instead half metallic for the InAs lattice constant (see inset of Fig. 6(b)).

In Table I, we present the total magnetic moment/cell for all the compounds considered. The half metallic ferromagnetic moments are marked in bold. Half metallicity may be monitored from the integer moments of the unit cell. It is seen that from all the combinations, VAs and $\mathrm{CrAs}$ are HMFs for both GaAs and InAs lattice constants. VSb and CrSb are also half metallic for GaSb and InSb lattice constants. VP, $\mathrm{CrP}$ and $\mathrm{MnP}$ are only HMFs at the InP lattice constant. This shows that the lattice constants and the resulting electronic structures play an important role in determining half metallicity, a finding that is also evident from Fig. 1. The moments listed in Table I are consistent with the calculated moments of 
$\mathrm{Cr}$ and $\mathrm{Mn}$ impurities in a GaAs host, if one neglects the effect of other defects e.g. As antisites 34]. To be more specific we note that $\mathrm{Mn}$ as an impurity in GaAs carries a magnetic moment of $4 \mu_{B} / \mathrm{Mn}$ atom in all theoretical first principles calculations based on local density approximation (LDA) that neglect other lattice defects such as As antisites. If one considers As antisite defects, or Mn interstitial atoms that couple antiferromagnetically to the Ga substituted Mn atoms, the effective moment becomes $\sim 2.4 \mu_{B} / \mathrm{Mn}$ atom in agreement with experimental data [34]. For the currently investigated materials only a limited set of experimental data have been published. However, CrAs has been synthesized in the zinc-blende structure and the magnetic moment was measured to be $3 \mu_{B} / \mathrm{Cr}$ atom $[9]$ in agreement with the present theory and previous calculations 9]. Since Mn has one extra electron in the spin up band, it is natural that the moment of MnAs is $4 \mu_{B} / \mathrm{Mn}$ atom, which is in agreement with the moment of dilute $\mathrm{Mn}$ in GaAs (if one neglects defects).

The explanation of the half metallicity and the integer moments is similar to that of a III-V diluted magnetic semiconductor. In a III-V DMS, a cation is removed by creating a vacancy containing 3 holes in the valence band. When a transition metal occupies this cation site, it donates 3 electrons to the anion dangling bonds to saturate the bonding. The remaining unpaired $d$ electrons of the TMs are responsible for the magnetic moments. Due to the large exchange splitting according to Hund's rule, the spin up and spin down d bands are separated from each other having different degree of hybridization with the valence bands. Hence, V, Cr and Mn have magnetic moments of 2,3 and $4 \mu_{B}$, respectively from the unpaired spin up d electrons. However, the widths of the $d$-bands are fairly large indicating a substantial amount of hybridization with the anion- $p$ orbitals.

TABLE I. Total magnetic moment/cell (in $\mu_{B}$ ). Each entry corresponds to the binary alloy consisting of a transition metal from the leftmost column and the group V element in the subsequent columns. The bulk semiconductors whose lattice constants are considered for each calculation are shown in suffices.

\begin{tabular}{|l|l|l|l|l|l|l|}
\hline \hline & $\mathrm{As}_{G a A s}$ & $\mathrm{Sb}_{G a S b}$ & $\mathrm{P}_{G a P}$ & $\mathrm{As}_{I n A s}$ & $\mathrm{Sb}_{I n S b}$ & $\mathrm{P}_{I n P}$ \\
\hline $\mathrm{Ti}$ & 0.00 & 0.00 & 0.01 & 0.29 & 0.00 & 0.04 \\
\hline $\mathrm{V}$ & $\mathbf{2 . 0 0}$ & $\mathbf{2 . 0 0}$ & 1.90 & $\mathbf{2 . 0 0}$ & $\mathbf{2 . 0 0}$ & $\mathbf{2 . 0 0}$ \\
\hline $\mathrm{Cr}$ & $\mathbf{3 . 0 0}$ & $\mathbf{3 . 0 0}$ & 2.74 & $\mathbf{3 . 0 0}$ & $\mathbf{3 . 0 0}$ & $\mathbf{3 . 0 0}$ \\
\hline $\mathrm{Mn}$ & 3.53 & 3.89 & 3.19 & $\mathbf{4 . 0 0}$ & 4.02 & $\mathbf{4 . 0 0}$ \\
\hline $\mathrm{Fe}$ & 2.36 & 2.69 & 1.06 & 3.20 & 3.26 & 3.15 \\
\hline $\mathrm{Co}$ & 0.02 & 0.23 & 0.00 & 1.15 & 1.39 & 0.94 \\
\hline $\mathrm{Ni}$ & 0.00 & 0.02 & 0.01 & 0.04 & 0.02 & 0.11 \\
\hline \hline
\end{tabular}

We also investigated compounds of $\mathrm{Ti}, \mathrm{Fe}, \mathrm{Co}$ and $\mathrm{Ni}$, and found that they do not show half metallic ferromagnetism (see Table I). Fe compounds have fairly big moments in the ferromagnetic phase except for $\mathrm{FeP}$ in the GaP lattice constant. CoSb in the InSb lattice constant

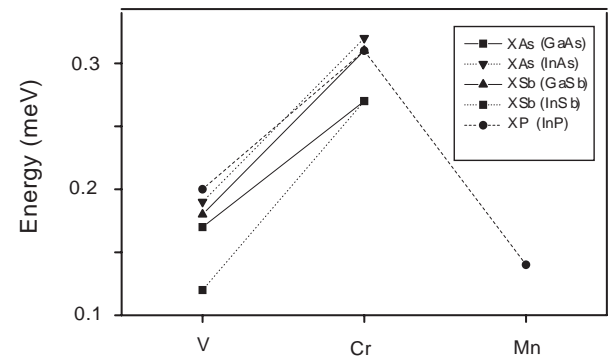

FIG. 2: Exchange interactions for $\mathrm{V}, \mathrm{Cr}$ and $\mathrm{Mn}$ compounds. Note that the data are given for lattice constants corresponding to GaAs, InAs, GaSb, InSb and InP substrates.

has the highest moment among the Co based compounds. $\mathrm{Ti}$ and $\mathrm{Ni}$ based compounds do not have prominent ferromagnetism. As only V, Cr and Mn based compounds show interesting and useful magnetic behavior, we will now concentrate on those compounds only.

TABLE II. Total energy differences in $\mathrm{eV} / \mathrm{Mn}$ atom $\left(\Delta E=E_{A F M}-E_{F M}\right)$ where $F M$ and $A F M$ are the ferromagnetic and antiferromagnetic arrangements between Mn atoms respectively. The symbol '-' is for the compositions where calculations were not performed.

\begin{tabular}{|l|l|l|l|l|l|}
\hline \hline & GaAs & InP & InAs & GaSb & InSb \\
\hline VAs & 0.17 & - & 0.19 & - & - \\
\hline CrAs & 0.27 & - & 0.32 & - & - \\
\hline VP & - & 0.2 & - & - & - \\
\hline CrP & - & 0.31 & - & - & - \\
\hline $\mathrm{MnP}$ & - & 0.14 & - & - & - \\
\hline VSb & - & - & - & 0.18 & 0.12 \\
\hline CrSb & - & - & - & 0.31 & 0.27 \\
\hline \hline
\end{tabular}

\section{B. Interatomic exchange interactions}

In this section, we compare the total energies of the HMFs found from our calculations, in the ferromagnetic (FM) and antiferromagnetic (AFM) phases. For briefness, calculations were only made for selected $\mathrm{V}, \mathrm{Cr}$ and Mn compounds (see Table II). In our calculations, we considered an 8 atom $(\mathrm{TM})_{4} \mathrm{X}_{4}$ cell in a layer geometry in the (001) direction with $2 \mathrm{TM}\left(\mathrm{TM}_{I}\right)$ atoms in the plane at $\mathrm{z}=0.0$ and $2 \mathrm{TM}$ atoms at $\mathrm{z}=\mathrm{a} / 2\left(\mathrm{TM}_{I I}\right)$, where $\mathrm{a}$ is the lattice parameter. Here TM and $\mathrm{X}$ indicate transition metal (V, $\mathrm{Cr}$ and $\mathrm{Mn}$ ) and the group $\mathrm{V}$ element (As, $\mathrm{P}$ and $\mathrm{Sb}$ ) respectively. $\mathrm{TM}_{I}$ and $\mathrm{TM}_{I I}$ atoms can couple ferromagnetically or antiferromagnetically. The energy differences are given in Table II and are also plotted in Fig. 2. The energy differences, which reflects the interatomic exchange coupling strengths are fairly large and are found to always stabilize ferromagnetism. From Fig. 2 and Table II, it is clear that the strongest exchange interaction is found for the $\mathrm{Cr}$ based 

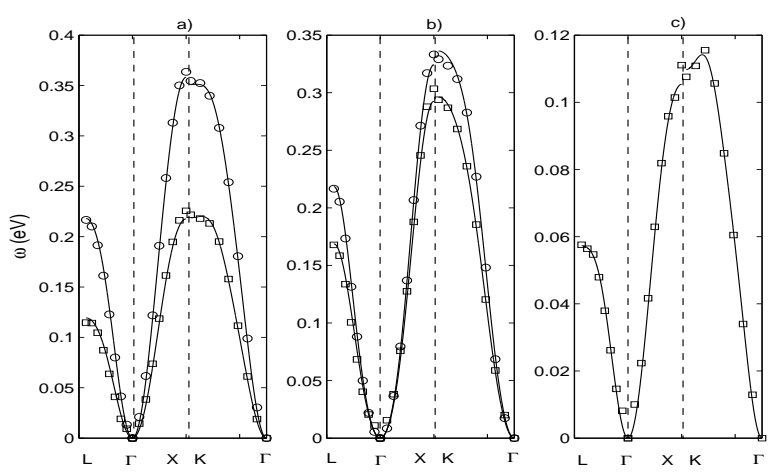

FIG. 3: Spin wave spectra along high-symmetry lines in the Brillouin zone of a) VAs b) CrAs c) MnAs. Circles are calculated values for the lattice constant of GaAs and squares are for the lattice constant of InAs. Solid lines represent the fitted data.

compound, where the energy difference is quite appreciable, indicating a high Curie temperature. An inspection of the DOSs for the FM and the AFM structures reveal that for the AFM structure, the DOS at the Fermi energy is non zero for both spin up and spin down bands whereas in the FM alignment, only spin up states exist at the Fermi level. This stabilizes the FM alignment, since the eigenvalue-sum in general lowers the total energy when electron states are removed from the Fermi energy.

In this section, we also present results of the calculations of critical temperatures using spin wave spectra and statistical mechanics. We have calculated $T_{c}$ for VAs, CrAs and MnAs for lattice constants of GaAs and InAs by extracting exchange interactions from calculated spin wave spectra, which subsequently were used in a classical Heisenberg Hamiltonian. In Fig. 3, the calculated

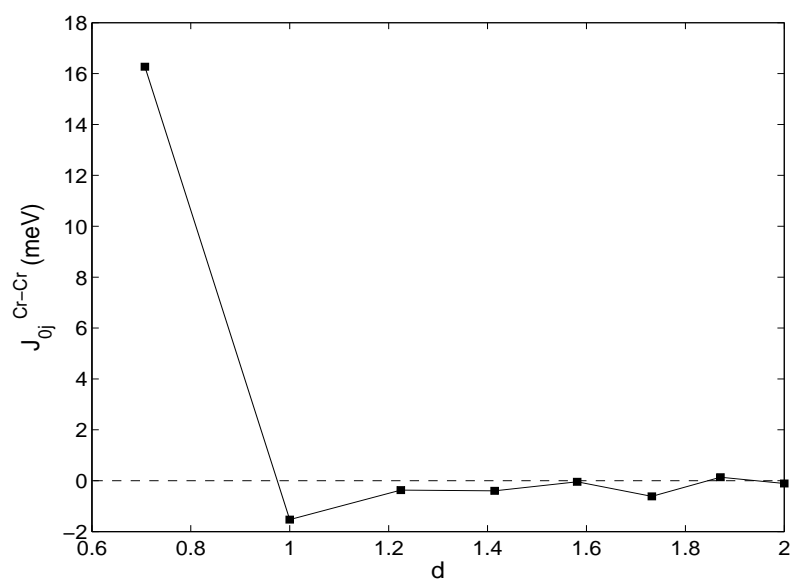

FIG. 4: Exchange interactions between the Cr atoms in CrAs as a function of $\mathrm{Cr}-\mathrm{Cr}$ distance (in units of GaAs lattice constant).

spin wave spectra for the systems are shown. MnAs in the lattice constant of GaAs was found to be antiferro- magnetic and is therefore not shown in the figure. The other systems were found to be ferromagnetic and half metallic with total magnetic moments of 2.0,3.0 and 4.0 $\mu_{B}$, respectively for VAs, CrAs and MnAs. In Table.III, calculated values of $T_{c}$ are shown. In the case of CrAs, a very high $T_{c}$ around $1000 \mathrm{~K}$ is estimated which could be very promising from an application point of view. $T_{c}^{M C}$ is smaller than $T_{c}^{M F A}$ due to the fact that fluctuations are absent in the latter. Normally, MC simulation gives $T_{c}$ values closer to experimental values than those obtained from mean field theory [26].

TABLE III. Calculated critical temperature of VAs, CrAs and MnAs from the MFA and MC simulations. Values at the left correspond to the lattice constant of GaAs and the right correspond to InAs.

\begin{tabular}{|c|c|c||c|c|}
\hline \hline & $T_{c}^{M F A}(K)$ & $T_{c}^{M C}(K)$ & $T_{c}^{M F A}(K)$ & $T_{c}^{M C}(K)$ \\
\hline VAs & 990 & 830 & 610 & 490 \\
\hline CrAs & 1320 & 980 & 1100 & 790 \\
\hline MnAs & - & - & 640 & 530 \\
\hline \hline
\end{tabular}

As is clear from Table III, the critical temperatures of VAs and CrAs are in general larger for the substrate with the smallest lattice constant (GaAs). Hence it is clear that the critical temperatures of VAs and CrAs follow the same trend as that of rare-earth systems, where in general a reduced lattice constant results in enhanced critical temperatures (as for instance measured by an applied external pressure 35]). In rare-earths this phenomenon is easily understood from the RKKY interaction, which is known to be responsible for the magnetic ordering in these elements. By analogy we can say that the presently studied materials (VAs and CrAs) have rare-earth like characteristics, in the sense that the exchange interaction between localized moments is mediated by more diffuse valence states.

In fig. 4 , we show the exchange interactions $\left(J_{i j}\right)$ in $\mathrm{CrAs}$ as a function of $\mathrm{Cr}-\mathrm{Cr}$ distance. It is clear that the first nearest neighbor exchange interaction is the dominant one and is ferromagnetic in nature. $J_{i j}$ decreases rapidly with increasing distance between $\mathrm{Cr}$ atoms. Further neighbor exchange interactions are antiferromagnetic in nature and are very weak. Similar kind of observation was reported by Sandratskii and Bruno [36] in the case of Mn doped GaAs.

\section{BAND STRUCTURE AND CALCULATION OF REFLECTANCE}

In Fig. 5(a) and (d), we show the band structures of MnAs in the InAs lattice constant for two symmetry directions viz. $\Gamma-\mathrm{L}$ and $\Gamma-\mathrm{X}$ directions respectively. In (b) and (c), we show the same for bulk InAs calculated for the experimental lattice parameter. Our calculation does not produce a gap at the $\Gamma$ point, so the top of 
the valence band and the bottom of the conduction band merge with each other. This is a well known limitation of using LDA or GGA within density functional theory. For MnAs, the spin up bands cross the Fermi level for the two symmetry directions shown the figure and also for other directions (omitted in the figure). There is gap in the spin down channel. As described above, the $e$ bands are dispersionless as they do not mix with others. The antibonding $t_{2}$ bands show large dispersion as they are hybridized strongly with the As $p$ bands.

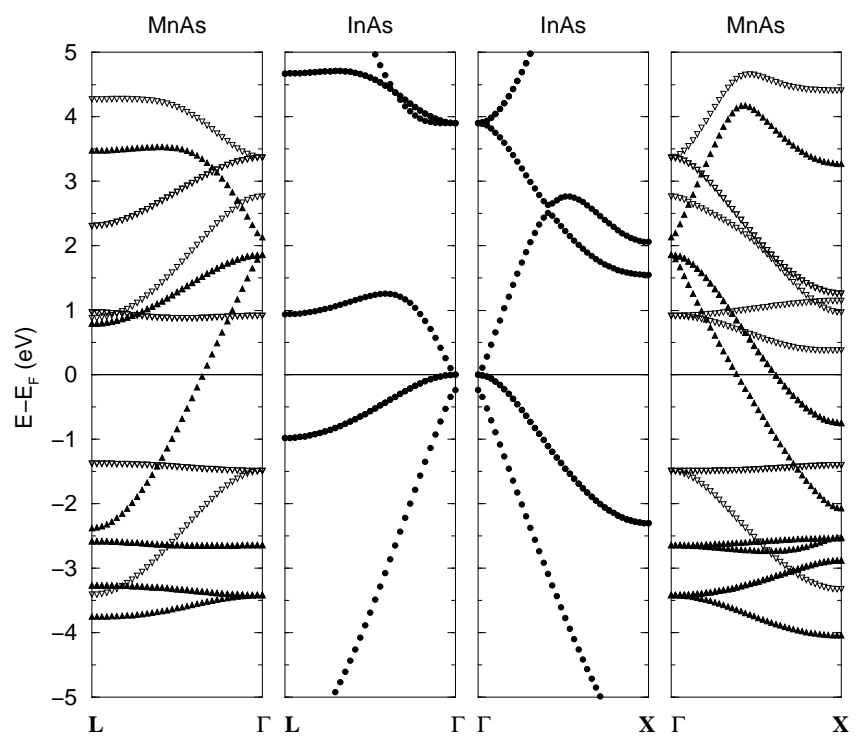

FIG. 5: Band structure of MnAs and InAs in two symmetry directions. For MnAs, the filled up triangles and empty down triangles indicate spin up and spin down bands respectively. The horizontal lines indicate the Fermi level of MnAs and the valence band maximum of InAs.

We now give a rough estimate of the reflectance and transmittance from a ferromagnetic metal/semiconductor interface from the band matching of the individual species. We use a simple free electron model, that was used also by Kilian and Victora 37. For an electron traveling through a one-dimensional potential step, the reflectance is given by

$$
R=\frac{\left(k_{f m}-k_{s c}\right)^{2}}{\left(k_{f m}+k_{s c}\right)^{2}}
$$

where $k_{f m}$ and $k_{s c}$ are the wave vectors in the ferromagnetic metal and semiconductor respectively. As the spin up bands are the only states available at the Fermi energy, we consider the following for the spin up electrons only. For InAs, as the conduction band minimum is at the $\Gamma$ point, the lowest energy levels available to accept spins from the metal are at the $\Gamma$ point. We calculate $R[100], R[110]$ and $R[111]$ for [100] $(\Gamma-X),[110](\Gamma-K)$ and $[111](\Gamma-L)$ directions, respectively. It is known from experiments that for e.g. an interface of $\mathrm{Au} / n$-InAs, the Fermi level is pinned $0.1 \mathrm{eV}$ above the conduction band minimum. We use this information in our model assuming that the properties of the metal do not alter strongly the band alignments. Also, the spins within $\left(k_{B} T \sim 0.025 \mathrm{eV}\right)$ of the MnAs Fermi level are the only ones available for transport properties. From this information we can calculate $k_{s c}$ for the three different directions. $k_{f m}$ for the three different directions are available from the band structures of MnAs directly. Then we calculated the reflectance from equation 1 . The calculated values are $R[100]=0.73, R[110]=0.89$ and $R[111]$ $=0.71$. This means that the transmissions in these directions will be rather small. However, one should bear in mind that in reality the interface is not ideal. There are interface states and different scattering mechanisms which can further decrease or increase the transmission through the interface. A full transport calculation taking into account a proper model of the interface could give a more realistic estimate, and our model should be viewed as an estimate only.

\section{STRUCTURAL STABILITY}

In this section, we present the relative structural stabilities of these compounds. For briefness, we choose to illustrate our conclusion only for 2 out of the 9 HMFs shown in Table I, i.e. CrSb and MnAs. The conclusions reached for these two compounds should be valid for all the HMFs listed in Table I. We compare the total energies of two crystal structures, hexagonal NiAs (that is the low temperature structure) and the zinc-blende (ZB) structure.
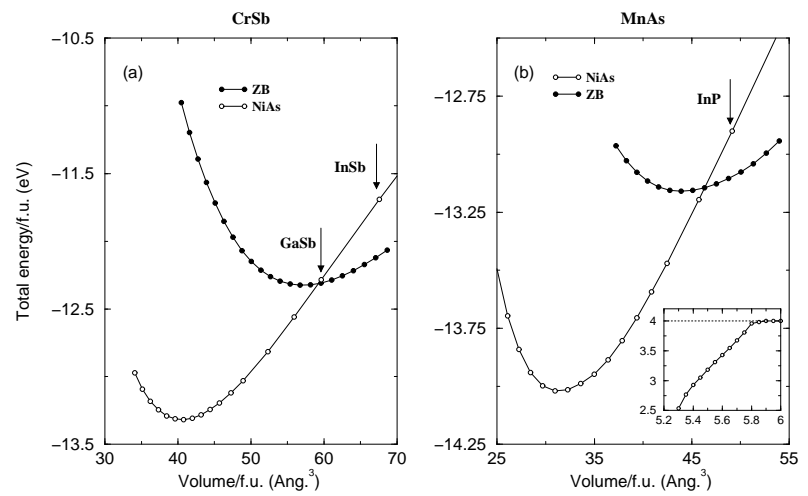

FIG. 6: Total energy vs. volume for (a) $\mathrm{CrSb}$ in the zincblende and the NiAs structure (b) MnAs in the zinc-blende and the NiAs structure with (inset) variation of total magnetic moment/cell with lattice constant for zinc-blende MnAs.

From Fig. 6, it is evident that for both MnAs and $\mathrm{CrSb}$, the NiAs structure is more stable than the $\mathrm{ZB}$ structure. Similar results were obtained for MnAs by Sanvito and Hill [11]. This is in accordance with naturally occurring compounds of $\mathrm{CrSb}$ and $\mathrm{MnAs}$ in the NiAs structure. However the total energy curves for the NiAs structure crosses the total energy curves of ZB structures for certain volumes. This occurs for a volume 
of $46.3 \AA^{3}$ /f.u. in MnAs and $59.6 \AA^{3}$ /f.u. in CrSb. Incidentally, these volumes are quite close to that of GaAs in case of ZB-MnAs and that of GaSb in case of ZB-CrSb compounds. For larger volumes, the ZB structure is more stable than the NiAs structure. Also, these compounds become half metallic ferromagnets according to the calculations. Therefore, ZB heterostructures of suitable combinations should be possible to use to obtain half metallic materials with a large critical temperature. Hopefully this fact can be a useful guideline for experimental work in trying to grow heterostructures for spin-electronics applications. Finally, the variation of magnetic moment of MnAs with the variation in lattice constant is shown in the inset of Fig. 6(b) where it is clear that it becomes half metallic only near the vicinity of a lattice constant of $6 \AA$.

The results presented above are in excellent agreement with the existing ones, wherever available. However, there are certain limitations of our approach. One of them is the underestimation of band gaps in semiconductors within the use of LDA or GGA. A more appropriate way is to use GW approximation [38] for these materials which corrects the band gaps extremely well. To our knowledge, no theoretical calculation exists for diluted magnetic semiconductors using this more complicated theory. The other issue is the effects of native defects which are always present in the growth processes. One should also take into account these effects to model a more realistic situation. But we believe that the results presented in this paper are quite significant as far as trends are concerned and also a guideline for future experiments.

\section{CONCLUSION}

In conclusion, we have identified a number of possible half metallic ferromagnets in the zinc-blende structure. Ferromagnetism has been shown to be more stable than an antiferromagnetic structure and exchange couplings are calculated to be sufficiently strong to produce high Curie temperatures, especially for $\mathrm{Cr}$ based compounds. We also give a parameter range in substrate lattice constants where MnAs and $\mathrm{CrSb}$ are expected to grow and to have half metallic properties. Finally, we predict that VAs grown at suitable conditions should be a ferromagnetic semiconductor with a large critical temperature. We hope to motivate experimental work in trying to grow such materials for future applications. From a theoretical point of view, the future scope of this work is to investigate the interface properties of these binary compounds and the zinc-blende semiconductors e.g. CrAs and GaAs. A detailed investigation including structural relaxations of these interfaces is in progress.

Acknowledgement 1 This work was done during the stay of one of the authors (B.S.) in Max-lab, Lund, Sweden. Support from the Swedish Science Foundation (VR), Swedish Foundation for Strategic Research (SSF) and the Göran Gustafsson foundation are acknowledged. Also, we acknowledge support from National Supercomputer Center (NSC).
[1] G. A. Prinz, Phys. today 48, 58 (1995); Science 282, 1660 (1998).

[2] R. A. de Groot, F. M. Mueller, P. G. van Engen and K. H. J. Buschow, Phys. Rev. Lett 50, 2024 (1983).

[3] Y. Ohno, D. K. Young, B. Beschoten, F. Matsukara, H. Ohno and D. D. Awschalom, Nature 402, 790 (1999).

[4] J. C. Egues, Phys. Rev. Lett. 80, 4578 (1998).

[5] R. Fiederling, M. Kelm, G. Reuscher, W. Ossau, G. Schmidt, A. Waag and L. W. Molenkamp, Nature 402, 787 (1999).

[6] G. Schmidt, L. W. Molenkamp, A. T. Filip and B. J. van Wees, Phys. Rev. B 62, 4790 (2000).

[7] H. Ohno, J. Magn. Magn. Mater. 200, 110 (1999).

[8] N. Theodoropoulou, A. F. Hebard, M. E. Overberg, C. R. Abernathy, S. J. Pearton, S. N. G. Chu and R. G. Wilson, Phys. Rev. Lett. 89, 107203 (2002).

[9] H. Akinaga, T. Manago and M. Shirai, Jpn. J. Appl. Phys. 39, L1118 (2000).

[10] J. H. Zhao, F. Matsukara, K. Takamura, E. Abe, D. Chiba and H. Ohno, Appl. Phys. Lett. 79, 2776 (2001).

[11] S. Sanvito and N. A. Hill, Phys. Rev. B 62, 15553 (2000).

[12] M. Shirai, Physica E 10, 143 (2001).

[13] A. Continenza, S. Picozzi, W. T. Geng and A. J. Freeman, Phys. Rev. B 64, 085204 (2001).

[14] I. Galanakis, Phys. Rev. B 66, 012406 (2002).
[15] G. Kresse and J. Hafner, Phys. Rev. B 47, RC558 (1993); G. Kresse and J. Furthmüller, Phys. Rev. B 54, 11169 (1996).

[16] P.E. Blöchl, Phys. Rev. B 50, 17953 (1994).

[17] S. Mirbt, B. Sanyal and P. Mohn, J. Phys. Condens. Matter 14, 3295 (2002).

[18] J. P. Perdew and Y. Yang, Phys. Rev. B 45, 13244 (1992).

[19] H. J. Monkhorst and J. D. Pack, Phys. Rev. B 13, 5188 (1976).

[20] A. Eichler, J. Hafner, J. Furthmüller and G. Kresse, Surface Science 346, 300 (1996).

[21] N. W. Ashcroft and N. D. Mermin, Solid State Physics, Saunders College Publishing, Harcourt College Publishers, 1976).

[22] We have done general tests to compare ASA and full potential (FP) results. They differ only by a few percent e.g. in the equilibrium lattice constant and magnetic moments. Values of exchange parameters are also very similar. Nearest neighbour exchange interaction parameters extracted from ASA calculations tally extremely well with those calculated from total energy differences in FP calculations.

[23] O. K. Andersen, Phys. Rev. B 12, 3060 (1975); O. K. Andersen and O. Jepsen, Phys. Rev. Lett. 53, 2571 (1984).

[24] H. L. Skriver, The LMTO Method (Springer-Verlag, 
1984)

[25] J. P. Perdew, K. Burke, and M. Ernzerhof, Phys. Rev. Lett. 77, 3865 (1996).

[26] N.M. Rosengaard and B. Johansson, Phys. Rev. B 55, 14975 (1997).

[27] S.V. Halilov, H. Eschrig, A.Y. Perlov and P.M. Oppeneer, Phys. Rev. B 58, 293 (1998).

[28] M. Pajda, J. Kudrnovsky, I. Turek, V. Drchal and P. Bruno, Phys. Rev. B 64, 174402 (2001).

[29] L.M. Sandratskii, Advances in Physics, 47 , 1 (1998).

[30] D.P. Landau and K. Binder, A Guide to Monte Carlo Simulations in Statistical Physics (Cambridge Univ. Press, 2000).

[31] S. Sanvito, P. Ordejon and N. A. Hill, Phys. Rev. B 63, 165206 (2001).

[32] T. C. Schultess and W. H. Butler, J. Appl. Phys. 89, 7021 (2001).
[33] Y.-J Zhao, W. T. Geng, K. T. Park and A. J. Freeman, Phys. Rev. B 64, 035207 (2001).

[34] P. A. Korzhavyi, I. A. Abrikosov, E. A. Smirnova, L. Bergqvist, P. Mohn, R. Mathieu, P. Svedlindh, J. Sadowski, E. I. Isaev, Yu. Kh. Vekhilov and O. Eriksson, Phys. Rev. Lett. 88, 187202 (2002).

[35] See articles in "Handbook on the physics and chemistry of the rare-earths", eds. K.A. Gschneidner and L. Eyring (North-Holland, Amsterdam, 1984).

[36] L. M. Sandratskii and P. Bruno, Phys. Rev. B 66, 134435 (2002).

[37] K. A. Kilian and R. H. Victora, J. Appl. Phys. 87, 7064 (2000).

[38] F. Aryasetiawan and O. Gunnarsson, Rep. Prog. Phys. 61, 237 (1998). 